Cinémas

Revue d'études cinématographiques

Journal of Film Studies

\title{
Médiations et captation de la réceptivité cinématographique posthollywoodienne
}

\section{Luciano Benvenuto}

Volume 2, numéro 2-3, printemps 1992

Cinéma et Réception

URI : https://id.erudit.org/iderudit/1001082ar

DOI : https://doi.org/10.7202/1001082ar

Aller au sommaire du numéro

Éditeur(s)

Cinémas

ISSN

1181-6945 (imprimé)

1705-6500 (numérique)

Découvrir la revue

Citer cet article

Benvenuto, L. (1992). Médiations et captation de la réceptivité cinématographique posthollywoodienne. Cinémas, 2(2-3), 148-170.

https://doi.org/10.7202/1001082ar

\section{Résumé de l'article}

Un des phénomènes majeurs liés à la réception filmique posthollywoodienne concerne les nouvelles « formes de médiation » qui s'exercent dans l'espace social de l'exploitation cinématographique contemporaine. Si les superproductions actuelles ont pu atteindre la dimension du " film-événement ", cela est dû en grande partie aux efforts conjugués de diverses forces de médiation qui captent et programment la réceptivité spectatorielle. Il faut donc comprendre, aujourd'hui, que derrière l'acte du spectateur d'aller voir un film en salle se dissimule, en fait, toute une série d'appels, d'incitations et d'envies provoquée par des médiateurs. Connaître le profil du public cinématographique est une chose, appréhender les formes de médiation qui l'interpellent en est une autre qu'il convient d'examiner. Pour ce faire, nous analysons quatre formes de médiation : 1 - séduction par médiation sociale, 2- séduction par médiation économique, 3- séduction par médiation psychologique, 4- séduction par relation interpersonnelle. 


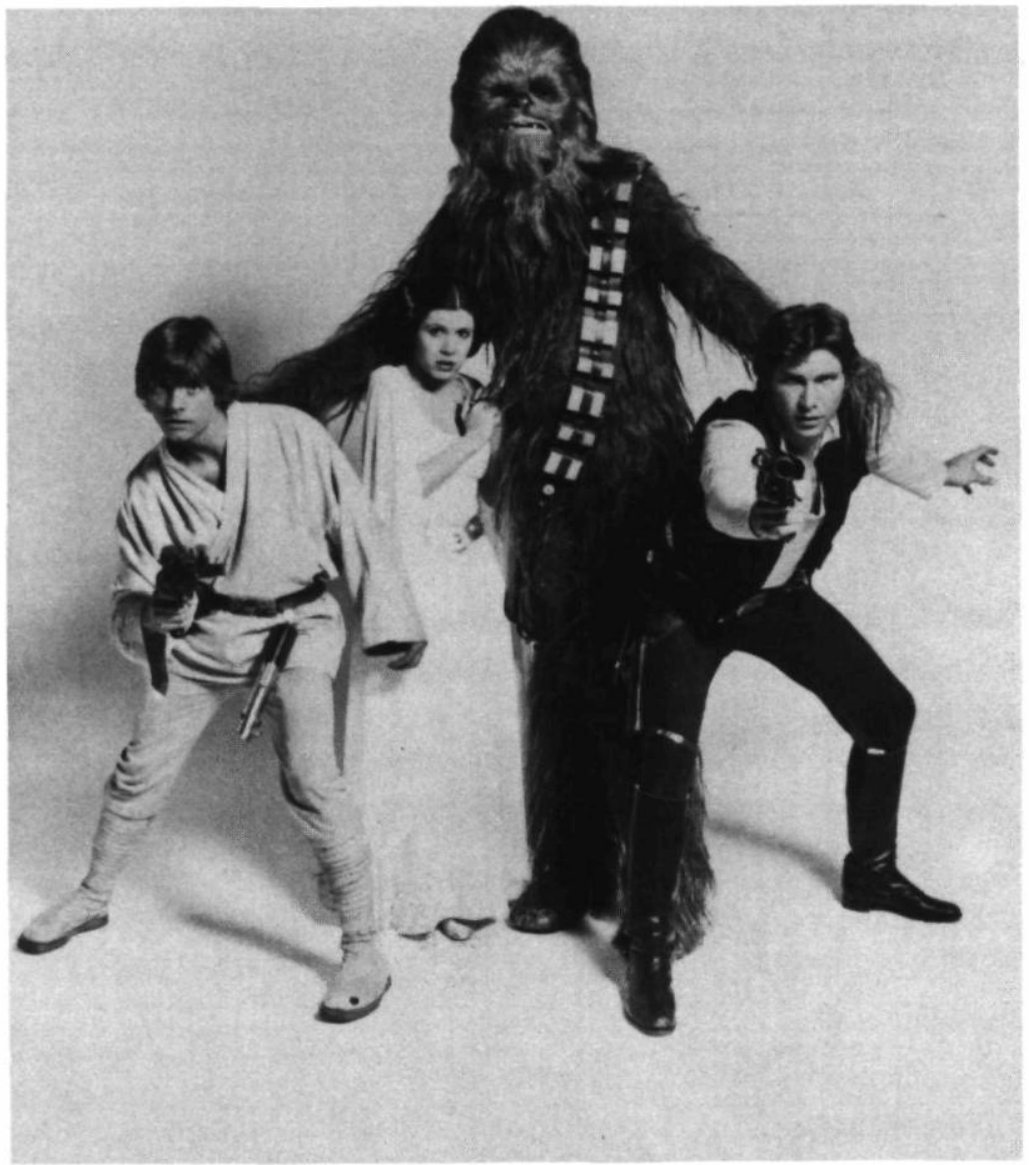

Star Wars de George Lucas (1977)

Coll.: Cinémathèque québécoise 


\title{
Médiations et captation de la réceptivité cinématographique posthollywoodienne
}

\section{Luciano Benvenuto}

\author{
RÉSUMÉ
}

Un des phénomènes majeurs liés à la réception filmique posthollywoodienne concerne les nouvelles «formes de médiation» qui s'exercent dans l'espace social de l'exploitation cinématographique contemporaine. Si les superproductions actuelles ont puatteindre la dimension du «film-événement», cela est dû en grande partie aux efforts conjugués de diverses forces de médiation qui captent et programment la réceptivité spectatorielle. Il faut donc comprendre, aujourd' hui, que derrière l'acte du spectateur d'aller voir un film en salle se dissimule, en fait, toute une série d'appels, d'incitations et d'envies provoquée par des médiateurs. Connaître le profil du public cinématographique est une chose, appréhender les formes de médiation qui l'interpellent en est une autre qu'il convient d'examiner. Pour ce faire, nous analysons quatre formes de médiation: 1- séduction par médiation sociale, 2- séduction par médiation économique, 3- séduction par médiation psychologique, 4- séduction par relation interpersonnelle.

\begin{abstract}
One of the most important aspects of film reception in the post-Hollywood period concerns the new «forms of mediation» that occupy the social space that surrounds contemporary cinema. Current superproductions have
\end{abstract}


now become film events in themselves, mainly as a result of the combined efforts of various forces of mediation which track and program spectatorial receptivity. Thus behind the spectator's act of film attendance, there lies a hidden series of appeals, urgings and desires unleashed by media forces. It is one thing to understand the profile of the film public, but it is another to grasp the forms of mediation by which that public has been interpellated. This is a topic which requires investigation. In this article, four forms of mediation are analyzed: seduction by social mediation, by economic mediation, by psychological mediation, and by interpersonal relationship.

Létude de la réceptivité, au cinéma comme dans les autres formes de communication, constitue un champ d'analyse assez récent. Après s'être longtemps préoccupé de la production des messages et de leur transmission, il est maintenant à la mode chez les chercheurs médiatiques d'essayer de comprendre comment s'exercent les mécanismes de la réceptivité. Mais, ici comme ailleurs, l'analyse du phénomène de la réceptivité englobe plusieurs facettes dont il est souvent difficile de démêler l'enchevêtrement interdisciplinaire, et ce d'autant plus lorsque l'angle d'observation des chercheurs n'est pas précisé avec soin. Pour éviter toute ambiguiité, la réceptivité au cinéma sera appréhendée ici uniquement d'un point de vue sociologique et seulement en fonction du contexte cinématographique posthollywoodien ${ }^{1}$. Plus précisément, notre analyse s'intéressera aux diverses formes de médiation s'exerçant aujourd'hui dans l'espace social de l'exploitation filmique dans le but de capter ou d'orienter la réceptivité du public cinématographique.

L'exploitation des films en salle est devenue en Amérique du Nord un secteur économique indépendant au sein de l'industrie du cinéma américain dès que les lois antitrust furent appliquées intégralement ${ }^{2}$. Libéré à partir de 1949 de la tutelle des Majors et de leurs pratiques commerciales hégémoniques, le secteur de l'exploitation cinématographique (basé sur la diffusion des films en salle) s'est donc soumis aux lois de l'offre et de la demande du marché libre. Or, cette nouvelle donnée traçait pour l'exploitation 
cinématographique une voie de développement économique beaucoup plus aléatoire et incertaine qui, au cours des décennies suivantes, allait devenir dramatique. Durant la crise du cinéma hollywoodien, qui culmina entre les années 68-73, le secteur de l'exploitation des films en salle fut en effet le secteur de l'industrie cinématographique le plus directement et le plus durement affecté par la chute de la fréquentation du public cinématographique.

Devant ce contexte de crise, on peut dire que le secteur de l'exploitation cinématographique a su assurer sa survie de deux façons: d'une part, en se réorganisant en fonction de la restructuration de l'industrie du cinéma posthollywoodien qui s'exerçait en amont et, d'autre part, en développant en aval de nouveaux mécanismes d'exploitation et de persuasion pour faire face à la réalité concurrentielle du marché audiovisuel. C'est ainsi que l'analyse de l'exploitation cinématographique posthollywoodienne se doit de considérer aujourd'hui trois phénomènes spécifiques, soit: 1) les nouvelles pratiques d'exploitation, 2) l'apparition d'un nouveau profil sociologique de spectateurs de cinéma et, enfin 3 ) les méthodes innovatrices en matière de «captation» et de "programmation» de la réceptivité cinématographique. Pour les besoins de cet article, nous insisterons sur le dernier thème, à savoir: les nouvelles «formes de médiation» apparues dans le champ cinématographique posthollywoodien.

$\mathrm{Si}$ les superproductions hollywoodiennes actuelles ont pu atteindre les dimensions du «film-monde» et du «film-événement», cela est dû en grande partie aux efforts conjugués de diverses forces de médiation. Il faut donc comprendre, aujourd'hui, que derrière l'acte du spectateur d'aller voir un film en salle se dissimule, en fait, toute une série d'appels, d'incitations et d'envies provoquée par des médiateurs. Connaître le profil du public cinématographique est une chose, appréhender les formes de médiation qui l'interpellent en est une autre plus capitale car, en dernière instance, le premier phénomène apparaît désormais de plus en plus déterminé par le second.

\section{La notion de médiation}

Par «médiation», nous entendons ce que les théories de la communication appellent les «relais d'opinion» ${ }^{3}$. En déplaçant 
l'analyse de la communication en fonction de ses rapports dialectiques à la culture de masse, au lieu d'en rester à la spécificité de ses aspects techniques, les modèles socioculturels appliqués à l'étude des média ont démontré que la diffusion du message entre émetteur et récepteur, loin de suivre un modèle mécanique et linéaire (émetteur-canal/message-récepteur), s'effectuait au contraire selon des schémas indirects, en parcourant des circuits relativement aléatoires où le rôle des relais d'opinion (ou des médiations) s'avère déterminant. La notion de médiation présuppose de fait l'existence de relais intermédiaires de diffusion dans le processus de communication. Ces relais ne sont pas bêtement des «courroies de transmission», mais constituent plutôt des lieux de traitement, de réorientation et de retransmission de l'information vers des publics cibles. Le cas du marketing, par exemple, représente une forme particulièrement efficace de médiation.

Dans la culture de masse, la médiation est un espace de programmation où des médiateurs ${ }^{4}$, qui en sont les stratèges et les agents exécutifs, s'appliquent à trouver des moyens de favoriser - ou plutôt d'inciter - l'accès du public à la réception des messages et des produits culturels. Lien indispensable aujourd'hui entre l'émetteur et le destinataire, les médiateurs sont les intercesseurs de l'industrie culturelle auprès du public. Ils représentent un réseau complexe d'influences socioculturelles qui rend leur fonction doublement «utilitaire». Économiquement d'abord, parce qu'en œuvrant à une meilleure visibilité des produits offerts, ils sont des embrayeurs entre la production culturelle et la consommation publique. Psychosocialement ensuite, parce que leurs objectifs visent à rejoindre le public dans le but de façonner son opinion et d'orienter ses goûts. De par ces pratiques sociales, les médiateurs deviennent ainsi des auxiliaires et des adjuvants du groupe industriel émetteur, ce qui, en dernière analyse, contribue à faire d'eux des instances supplémentaires de légitimation. On peut donc considérer, à juste titre, l'usage spécifique de diverses formes de médiation et leur organisation d'ensemble comme une stratégie de captation et de programmation du public.

Dans l'institution cinématographique, la médiation loge au 
centre du rapport conflictuel qu'entretiennent, d'une part, le groupe émetteur de l'industrie (producteurs-créateurs-professionnels) et, d'autre part, les sous-groupes de spectateurs aux valeurs et aux aspirations diversifiées. Malgré le poids du système financier, industriel et commercial exercé par les Majors, l'institution posthollywoodienne n'est aucunement assurée de la fidélité de son (jeune) public assidu, et ce d'autant plus que celui-ci fait face depuis quelques années à une très forte sollicitation de la part des nouveaux média audiovisuels. A défaut de pouvoir contrôler cette demande instable et fuyante, l'industrie posthollywoodienne, en tant que source émettrice, s'est donc appliquée, par l'intermédiaire de médiateurs chevronnés, à développer et à raffiner des techniques de persuasion afin d'en arriver à infléchir à la fois la décision et le choix du public spectateur. La solution trouvée pourrait se résumer au leitmotiv suivant: «Susciter le film-événement.»

Cette stratégie spécifique de captation spectatorielle s'articule, nous semble-t-il, autour de quatre modes particuliers de médiation qui, pour être simples, parfois complexes voire occultes, se sont avérés très efficaces comme en fait foi la très grande popularité obtenue par les films posthollywoodiens sur la scène cinématographique mondiale. La séduction nous paraît être le fil conducteur qui unifie ces pratiques de médiation pour attirer les spectateurs vers les salles de cinéma. Par souci de limitation, nous ferons ici un survol de ces quatre modes de séduction, par médiations sociale, économique, psychologique et par relation interpersonnelle, en exposant les points marquants.

\section{Séduction par médiation sociale}

Ce type de médiation concerne spécifiquement toutes les activités de publicité et de promotion rattachées au lancement massif des films ${ }^{5}$. Il n'est pas rare que la carrière d'un film soit conditionnée par la réussite ou non de sa campagne publicitaire. La conviction «qu'un film vaut ce que vaut son lancement» fait consensus dans le milieu posthollywoodien.

C'est ainsi que pour susciter un succès de curiosité capable d'attirer un grand nombre de spectateurs, il est nécessaire de maximiser la force de frappe publicitaire pour créer «l'événe- 
ment». La publicité cinématographique utilise à cette fin les autres média (affiche, presse, radio, télévision) qui, ensemble, véhiculent avec redondance une information séductrice dont les connotations affectives relancent beaucoup plus les aspects commerciaux du film que ses caractéristiques particulières. La recherche systématique de l'insolite, de l'anecdotique, du sensationnalisme accentue l'importance de l'emballage au détriment du contenu. L'image choc et l'effet accrocheur prédominent ${ }^{6}$. Comme les films sont calibrés pour une clientèle jeune, le contenu publicitaire doit forcément l'être aussi. En résumé, le but de «l'art publicitaire» en matière filmique est de proposer «à chacun des individus composant la cible de monter lui-même un film dans son imagination à partir des informations et des messages publicitaires qui l'agressent, le charment et parfois le fascinent, pour faire de lui un spectateur.» ${ }^{7}$

Depuis une dizaine d'années, certains média comme la presse et la télévision, toutes tendances culturelles et politiques confondues, réservent un créneau beaucoup plus large aux activités cinématographiques ${ }^{8}$. Les médiateurs sociaux tels les journalistes, les critiques, les animateurs et les analystes, en prenant soin de commenter, de nuancer ou de personnaliser l'information sur les films, moussent en fait leur visibilité et deviennent de la sorte des «faiseurs d'opinion» ${ }^{9}$. Et Philippe J. Maarek de souligner, bien à propos, que leur critique est généralement «bien plus une critique des éléments de commercialité qu'une information neutre sur les films. (...) Le contenu des critiques (...) se rattache en réalité fort étroitement à la logique de la consommation» (p. 190). Seules les revues spécialisées, de plus en plus variées mais au tirage limité, évitent cet écueil en s'adressant surtout, il faut le dire, à une «clientèle cinéphilique» réduite.

Le tableau suivant offre une synthèse des niveaux de communication atteints par les différentes formes de médiations publicitaires en regard du cinéma. Malgré la diversité des médiateurs et la pluralité de leurs actions, il faut noter que la valorisation économique des films-événements amène nécessairement à privilégier la médiation de masse. 
Figure 1

\section{Niveau/Médium/Type de médiation}

\section{Niveau de communication}

1. De masse

2. De classe

3. De groupe

4. De groupe restreint

5. Interpersonnelle sans Médiation

\author{
Moyen de \\ communication
}

Affiches; T.V., Radio, Grande Presse

Grande Presse

Presse

spécialisée

Pressse locale, Correspondance

Téléphone, Conversation

\section{Type d'actions des} médiateurs
Critique classique dans les quotidiens et les hebdos

Critique de revues spécialisées

Animation, Ateliers, Festivals, Ciné-Clubs

Propagande orale, Bouche à oreille, Rumeur

\section{Séduction par médiation économique}

En complément au «carnaval publicitaire» de lancement d'un film posthollywoodien auquel personne ne peut échapper, les stratèges en marketing ont élaboré deux formes particulières de médiation commerciale liées spécifiquement au film: les produits dérivés et le couplage.

L'invasion tout-terrain semble être devenue la norme de la 
nouvelle stratégie de distribution des produits culturels de masse. La superproduction ne se conçoit plus sans une multitude de produits dérivés qui intègrent d'une manière croissante les films au circuit des marchandises. Chaque film à grand succès tend à laisser dans son sillage un arsenal de marchandises et d'objets rappelant et exploitant les personnages, les costumes et les gadgets représentatifs du film. L'effigie de Superman a généré plus de 1500 produits différents fabriqués par 160 firmes $^{10}$. Pour des films comme E. T., Star Wars, Raiders of the Lost Ark, Star Trek, et plus récemment Ghostbusters ou Batman, il est facile de répertorier une centaine de produits qui leur sont liés et qui ont envahi les moindres recoins de la vie quotidienne ${ }^{11}$. Selon A. Mattelart, de 1978 à 1982 (années marquées notamment par la sortie des films Star Wars et E.T.), «le chiffre d'affaires réalisé grâce aux produits vendus sous le label des personnages ou des thèmes de films a triplé, passant de 6,6 à 20,6 milliards de dollars» (p. 145). Des cinéastes comme Lucas et Spielberg, loin d'avoir l'innocence, la candeur ou l'âme d'enfant que leurs films laissent suggérer, sont en réalité de véritables «calculateurs». Aux dires de Lucas, en même temps qu'il préparait Star Wars, il faisait une étude de marché pour tous les produits dérivés du film susceptibles d'être commercialisés (T-shirts, autocollants, disques, modèles réduits, figurines, bande dessinée, etc.) et dont il s'assurait bien de garder tous les droits exclusifs ${ }^{12}$. La musique de film représente aussi un produit dérivé important et fort lucratif notamment pour des compagnies comme Warner, MCA/Universal ou Columbia qui possèdent chacune leur propre division d'enregistrement/diffusion musicale (disques, cassettes, disques-laser). À ce chapitre, la recette des «ventes musicales» s'avère proportionnelle à la popularité des films.

D'autre part, une nouvelle action promotionnelle, appelée le couplage ou tie-ins ${ }^{13}$, a vu le jour récemment. Elle consiste à mettre en conjonction, pour une période limitée, la sortie d'un film à succès avec une campagne de promotion lancée soit par des chaînes de restauration rapide, des compagnies de boissons gazeuses ou par toute autre industrie de produits matériels. Ces opérations de couplage permettent, par exemple, à Pepsi-Cola de mousser la consommation d'un film «Y» en offrant des réductions 
sur le prix d'entrée du dit film. En contrepartie le Major remettra à l'achat de la vidéocassette du film un rabais sur la consommation des produits de Pepsi-Cola. Il n'est pas rare non plus de voir certaines chaînes de restauration remettre des billets gratuits (pour un film ou dans une salle en particulier) aux clients qui ont acheté de ses produits pour plus d'un certain montant ${ }^{14}$. L'interchangeabilité de la valeur du film par celle d'un produit de consommation (boisson gazeuse, repas, etc.) ne fait que souligner de nouveau ici, d'une façon à la fois métaphorique et ironique, le caractère commercial de la marchandise-film qui l'unit étroitement aujourd'hui à la culture et à l'économie. Comme le disait déjà Guy Debord en 1971: «La culture devenue intégralement marchandise doit aussi devenir la marchandise vedette de la société spectaculaire» (p. 193).

La pratique des produits dérivés et couplés constitue un mode de médiation économique efficace. Ces produits auxiliaires sont autant de signes marqués qui s'immiscent partout dans l'environnement social et culturel et qui interpellent insidieusement la conscience du public pour lui rappeler constamment l'existence d'un film particulier. Ce type de médiation économique s'articule sur un mouvement de concentration au plan symbolique et entraîne un effet de programmation/persuasion quasi-subliminal de l'opinion: le jouet, le disque, le gadget renvoyant au film à succès, «relancent ainsi continuellement une chaîne de sens, dans un immense syndrome de la répétition» (Mattelart et al.,p. 178). Seul l'appareil industrialo-culturel américain a cette possibilité et cette capacité de lancer, d'inscrire et d'étendre cette dimension symbolique dans le circuit des marchandises. Et le phénomène ne peut que s'amplifier avec les nouvelles technologies qui puisent déjà allégrement dans ce vaste arsenal symbolique (jeux vidéo, vidéoclip, etc.).

\section{Séduction par médiation psychologique}

Cette forme de médiation a pour objectif la programmation psychologique des spectateurs/consommateurs. Deux phénomènes majeurs y contribuent.

D'abord, la présence massive de ce qu'on nomme couramment en anglais les sequals et que nous traduirons, à défaut d'un meilleur 
terme, par «suites filmées», «séries filmées» ou «films à suite(s)» ${ }^{15}$. Cet élément est un des phénomènes les plus caractéristiques du cinéma posthollywoodien ${ }^{16}$. «L'effet-série» exerce une force d'attraction psychologique irrésistible en programmant chez le spectateur une structure positive de réceptivité par anticipation. Voyons comment le fonctionnement des «suites filmées» peut avoir un impact sur le choix filmique et la fréquentation des spectateurs.

La pratique avouée des «films à suites» correspond à une spécificité intrinsèque du modèle posthollywoodien. À preuve, des 32 meilleurs succès commerciaux de la période 1976-1985 ${ }^{17}$, plus des deux tiers d'entre eux (21 films) s'avèrent être soit des suites, soit les films initiateurs d'une «série filmée» proprement dite. De plus, si l'on considère que, parmi la liste des 15 plus grands hits de l'histoire d'Hollywood, seuls les films E.T. et Tootsie échappent à cette règle de la répétition, force est de reconnaître la popularité de cette formule, non seulement au sein de l'industrie mais également auprès des spectateurs ${ }^{18}$.

Avec la présence aussi tangible des «films à suites», on a l'impression que la loi de la répétition s'est donné aujourd'hui une nouvelle légitimité, à la fois socio-économique et psychologi$q^{19}$. Le symptôme révélateur de cette nouvelle légitimité est qu'au lieu de se dissimuler, de ne pas se laisser voir comme autrefois, la répétition sérielle s'exhibe fièrement à l'heure actuelle dans la numérotation successive des films au fur et à mesure de leurs sorties sur nos écrans. Suites sans fin, dont l'ironie est poussée à son comble dans le film Back to The Future II où une salle de cinéma du $\mathrm{XXI}^{\mathrm{e}}$ siècle met à l'affiche le film «holographique»Jaws $X$, réalisé par nul autre que Max Spielberg, le fils de Steven!!! La «suite filmique» donne dans l'autoparodie intertextuelle.

La pratique des «films à suites» est le résultat d'une logique économique qui consiste à mettre systématiquement en série des films qui ont connu de très grands succès commerciaux. L'application du principe se révèle dans un besoin impérieux de donner une suite à un film original, en reproduisant selon un moule identique les principaux facteurs empiriques qui ont rendu possible la réussite de celui-ci au box-office. La loi des «séries 
filmées» est donc dominée par une quête incessante de produire le plus grand succès possible à partir d'un filon commercial déjà éprouvé. Ce mécanisme est de nature essentiellement conservatrice, car il tend à réduire les incertitudes en balisant la créativité ${ }^{20}$. Plutôt que de risquer un échec en tentant d'attirer le public par la nouveauté, on préfère en général essayer de retrouver une formule, une recette, qui a précédemment obtenu un certain succès. Pour faire corps dans une «série filmée», les films en question doivent obéir à certaines conditions, à certains facteurs clefs qui leur procurent une unité d'ensemble, une homogénéité.

A- D'abord, ils doivent obligatoirement avoir entre eux certains dénominateurs communs dictés par le film original de la série, tels le même «genre» et le même contenu thématique. Cette uniformité primaire permet d'homogénéiser à la fois le traitement formel et le traitement narratif de la diégèse de la série.

B- Le second facteur est l'emploi des mêmes personnages, incarnés si possible par les mêmes acteurs. La star est un élément essentiel à une série, elle favorise et accélère le processus d'identification du spectateur. Un acteur bien coté est un facteur supplémentaire d'accroissement potentiel du succès recherché. Des séries comme Star Wars, Rocky, Star Trek utilisent les mêmes acteurs/personnages principaux, et parfois même secondaires. Indiana Jones, Superman, Airport focalisent sur un personnage unique mais toujours joué par la même star (H. Ford, C. Reeves, G. Kennedy), tandis que des séries comme Jaws, Omen, Halloween, Amityville ou Friday the 13th offrent moins d'homogénéité en variant les acteurs/personnages d'un film à l'autre.

C- Un même réalisateur/concepteur constitue aussi un facteur humain qui peut contribuer potentiellement à l'homogénéité de la série, par ex.: Star Wars (Lucas), Indiana Jones (Spielberg), Rocky (Stallone).

D- Finalement, la symbiose entre les suites d'une série sera d'autant plus accentuée si celles-ci manifestent entre elles un rapport étroit de consécution/conséquence. Autrement dit, des relations temporelles et causales entre les films assurent à la série filmée un très fort caractère d'unité qui risque de mobiliser encore plus l'attention du spectateur. En effet, le spectateur se sent sollicité à voir le deuxième film de la série car celui-ci est la 
réponse directe aux questions soulevées par le premier et, en même temps, le prélude aux événements de la troisième partie. Ainsi, bien que l'on retrouve des séries où les suites sont autonomes les unes face aux autres sans lien de consécution/conséquence entre elles (Indiana Jones, Superman, Jaws, Halloween, Airport, Amityville), il y en a d'autres qui, au contraire, sont fortement liées par un rapport temporel ou causal (Star Wars, Rocky, Star Trek, Alien).

Si nous accordons une importance particulière à ces quatre types de facteurs d'homogénéisation «des films à suites», c'est que le cumul ou non de ces facteurs au sein d'une série est en étroite corrélation avec le succès ou l'échec commercial de cette série $^{21}$.

Mais les «suites filmées» sont aussi le résultat d'une logique économique qui s'appuie sur une programmation psychologique. En dernière analyse, il apparaît en effet que le choix d'une suite filmique est en grande partie déterminé par l'engouement du public lors de la réception du film précédent de la «série filmée». Ce qui tend à prouver la fidélité d'un public satisfait d'un produit qui lui a été proposé même si celui-ci est répétitif dans son ensemble. On assiste à ce que Pierre Sorlin appelle «un effet cumulatif: la reprise d'un personnage, d'un sujet, d'une situation, loin de fatiguer les spectateurs, produit chez eux un phénomène d'accoutumance (...). Le plaisir provoqué par la répétition explique, en partie, le succès de certains films ou de certaines séries» (p. 125). Selon nos propres études, l'opération reste très rentable uniquement dans le cas, et dans ce cas uniquement, où une «série filmée» homogénéise ses suites filmiques ${ }^{22}$.

La loi des séries entraîne un «effet-série» de nature psychologique sur le public cinématographique. Les «suites filmées» alimentent le désir spectatoriel de retrouver des affects similaires à ceux ressentis lors des précédentes projections filmiques de la série. Le plaisir entretenu et renouvelé par «l'effet-série» conditionne en un sens le désir du spectateur.

Avec les «films à suites», l'offre se lance au-devant de la demande/consommation, qu'elle a elle-même anticipée et entretenue à la suite de la présentation du (ou des) premier(s) film(s) de la série. La force de persuasion des «séries filmées» est attribuable 
avant tout à la spécificité d'un produit qui contient en lui-même sa propre campagne promotionnelle; chaque suite suscite chez le spectateur consentant le désir de retrouver et de reconnaître des émotions déjà ressenties: non la recherche d'une nouveauté, mais la confirmation d'une habitude, d'un genre, d'un schéma narratif, de la répétition des personnages et des acteurs. L'autopromotion dans laquelle baigne une «série filmée» de superproductions performantes facilite leur succès commercial, suscite l'émergence du film-événement et, par effet d'entraînement développe une captation du jeune public cinématographique contemporain.

Contrairement à des auteurs comme Robert B. Ray, qui voit dans cette tendance marquée d'Hollywood pour les séries le signe d'une incertitude croissante de l'industrie à renouveler ses structures ainsi que le symptôme d'une absence soudaine de créativité et de renouvellement ${ }^{23}$, nous pensons que ce choix est délibéré et conforme à une stratégie transnationale de l'audiovisuel américain. Les «séries filmées», principalement composées de superproductions, tendent à régler rigoureusement la production de sens pour le spectateur en prescrivant «l'usage le plus universel ou la lecture la plus prévisible» des signes. «La série tend à niveler les processus de communication avec des spectateurs inscrits dans des espaces culturels différents» (Mattelart et al., p. 184). C'est ce qui fait précisément de la série «la matrice de production et de programmation la plus conforme à un modèle de conquête des marchés - intérieurs et extérieurs» (Mattelart et al., p. 190).

La seconde forme de médiation psychologique procède d'une démarche différente. Au lieu de chercher «à façonner» le goût du public, elle vise plutôt à cerner ses attentes en stimulant ses réactions. Les progrès obtenus dans la connaissance des comportements psychiques et cognitifs des spectateurs sont attribuables entre autre à l'usage systématique des «projections-tests», appelées communément dans le milieu sneakpreviews. Le film qu'on veut tester est présenté en salle à un public représentatif de spectateurs qui se sont déplacés pour en voir un autre, puis, l'étonnement passé, on tente par la suite d'observer attentivement leurs réactions. Les informations ainsi collectées (l'usage d'un questionnaire est fréquent) seront utilisées pour le remontage du film (si nécessaire) en accord avec les «goûts» du moment ${ }^{24}$. 
Plusieurs films, dont notamment ceux de F. Coppola (Apocalypse Now, One from the Heart), ont échoué lamentablement à ces tests spectatoriels. À ce stade crucial, les déboires encourus sont toujours lourds de conséquences financières: remontage ou abandon du film, ajout de séquences, modification de la stratégie de lancement, réduction du budget publicitaire, etc. Par contre, une réponse enthousiaste du public signifie, à bien des égards, la fin des angoisses et l'espoir d'un (certain) succès commercial du film.

Le cumul des informations recueillies lors des projections-tests enrichit, à moyen terme, la connaissance empirique des cinéastes au sujet des éléments et des mécanismes cinématographiques (récit, narration, histoire, rythme, effets visuels, etc.) qui suscitent et entretiennent le plaisir filmique des spectateurs. L'expertise ainsi acquise leur permet de «calibrer sur mesure» les films dès leur conception et de travailler à des patterns qui favoriseront une meilleure réceptivité de leurs œuvres.

Les médiations psychologiques, telles la projection-test, l'effetsérie ou toute autre technique spéculative d'enquête, confèrent ainsi à l'industrie hollywoodienne une efficacité accrue dans le développement de produits filmiques pondérés et ajustés aux désirs dominants. La modélisation et la programmation spectatorielles qui en découlent, réduisent à coup sûr la part du risque qui a toujours fait de l'industrie cinématographique une industrie plus hasardeuse et moins fiable qu'une autre. L'«aléatoire» n'est pas supprimé pour autant. L'industrie du cinéma commercial reste hautement spéculative, ce qui l'oblige à continuer de traquer inlassablement son public et de sonder ses goûts, afin d'éviter de produire des films qui iraient à l'encontre soit de ses attentes, soit de ses compétences d'écoute filmique. Le dilemme hollywoodien, entre le besoin de reconduire les codes établis et la nécessité d'innover, demeure donc entier.

\section{Séduction par relation interpersonnelle}

Dans le processus de communication de masse, il est généralement admis que les «relations interpersonnelles» jouent un rôle important dans la perception et la sélection des messages. C'est ainsi qu'après avoir analysé plusieurs résultats d'enquêtes, Katz 
et Lazarsfeld ont été amenés à faire ce constat et à formuler la théorie du «Two-Step Flow of Communication» ${ }^{25}$. Cette théorie propose un schéma de communication basé sur un mécanisme de transmission à deux niveaux. Lés mass média influencent d'abord certains «leaders d'opinion» informels, lesquels, à leur tour, influencent d'autres individus qui gravitent dans leur groupe de référence ${ }^{26}$. Autrement dit, une grande partie du public n'accède à l'information transmise que si celle-ci est d'abord répercutée par les «leaders d'opinion» informels propres à des groupes intermédiaires beaucoup plus réduits ${ }^{27}$. Le leader est celui qui reçoit et véhicule le maximum de communication, tant fonctionnelle qu'émotionnelle, à l'ensemble des autres membres du groupe. Son rôle social est donc primordial puisque «ses opinions vont servir de modèles et être reproduites, recopiées, répétées dans un processus de recrutement» (Moles, p. 436).

Il y a des leaders d'opinion pour la mode, les affaires, les idées politiques, comme pour le cinéma. Aujourd'hui, par leur dominance au sein du public cinématographique, les jeunes occupent, aux États-Unis comme ailleurs, cette fonction privilégiée de leader d'opinion dans le domaine du cinéma. Ils sont tous concernés par le film (leur loisir principal) et ce sont eux qui en voient le plus grand nombre dans les salles. Parce qu'ils ont une vie sociale active, fréquentent les institutions scolaires et vivent encore en famille, ils jouissent d'un grand potentiel d'influence dans leur milieu qui ne peut être que favorable pour l'activité cinématographique posthollywoodienne ${ }^{28}$.

Mais, dans ce processus de choix et de décision en matière cinématographique, la meilleure forme de persuasion interpersonnelle reste encore le bouche-à-oreille. Sous-produit de la communication sociale, la «rumeur», ainsi dénommée en psychologie sociale ${ }^{29}$, exerce un rôle important dans la diffusion de la culture, puisque son caractère incitatif repose sur un réseau de relations privées (amis, famille, milieu de travail). Un film à l'affiche qui ne bénéficie pas du bouche-à-oreille est assuré de disparaître rapidement. La «rumeur» permet, en effet, non seulement d'inciter d'autres spectateurs potentiels à aller voir concrètement les films, mais apporte aussi un complément d'information à ceux dont l'opinion serait plus ou moins arrêtée. Les 
films commerciaux bénéficiant d'un matraquage publicitaire intense ont ainsi plus de chance, eu égard à leur qualité, de s'infiltrer dans la rumeur de par le prestige qu'ils occasionnent. Les gens recherchent les films commerciaux pour se divertir, lesquels deviennent, après-coup, des sujets de discussion. Avec la rumeur, le public rencontre ainsi l'œuvre filmique sans avoir besoin d'être aidé, conseillé ou incité, ce qui tend à rendre inefficace ou superflu tout travail des médiateurs. La relation interpersonnelle accroît l'autonomie des sujets/spectateurs en développant leurs capacités de résistance au conditionnement médiatique et concurrence ainsi l'influence des moyens de communication de masse.

\section{En guise de conclusion}

De l'analyse des rapports médiatisés entre la production et la consommation posthollywoodiennes, on retiendra, en conclusion, les constatations suivantes.

Le soutien et le développement des médiations sont devenus aujourd'hui des facteurs essentiels pour accroître l'efficacité de la communication cinématographique. À cet effet, le système posthollywoodien a développé ses moyens d'action et engendré ses propres stratégies de captation du public cinématographique. En tant que source émettrice, il s'appuie directement sur des médiateurs institutionnels (publicité-affiches, presse, radio, TV, critique, animateurs, etc.) mais cherche aussi à courtiser de nouveaux espaces de médiations non institutionnels (produits dérivés, projection-test, effet-série, leader d'opinion, rumeur) comme catalyseurs d'une diffusion plus vaste. La conjonction de ces diverses formes de médiation crée l'événement-spectacle, condition indispensable au succès commercial des superproductions filmiques. Cette médiation globale renvoie bien entendu au système culturel et, par-delà, au système social et économique tout entier.

Dans l'ensemble de la médiation cinématographique, le destinataire - que ce soit le public, un groupe ou un individu — est fort actif. Selon ses besoins et ses aspirations en matière cinématographique, il prélève et sélectionne parmi les médiateurs intermédiaires présents dans son environnement celui ou ceux qui lui offrent le plus d'intérêt et les meilleures propositions en regard de 
ce qu'il recherche. Ainsi, du point de vue du récepteur, les médiateurs apparaissent, à prime abord, comme des agents de diffusion à son service.

Or, ces médiateurs ont élaboré leur propre compréhension subjective et motivée du message à transmettre. Ils véhiculent donc tout bonnement cette information, et, par leur avis, incitent le récepteur à s'y conformer. Ainsi, la sélection du destinataire est influencée par un discours séducteur sur le discours dont font l'objet les films. Il s'ensuit d'ailleurs une prédisposition à croire uniquement ce que le médiateur a compris, plutôt que de rechercher une interprétation personnelle. «Manipuler par séduction» l'opinion publique afin d'orienter son choix dans la sélection des films à consommer, tel est le but des opérations de médiation.

La «soumission docile» d'une grande partie du public spectateur aux impulsions des médiateurs n'est pas sans conséquences sur la nature socio-économique de l'activité cinématographique. En effet, il faut hélas remarquer que la mission de diffusion, de dialogue, d'éveil critique, d'éducation, normalement liée au travail des médiateurs, ne peut s'exercer convenablement parce qu'ils sont eux-mêmes inféodés ou tributaires des émetteurs, c'est-à-dire des groupes financiers qui contrôlent l'industrie cinématographique posthollywoodienne. Au sein de ce système axé sur le profit, le médiateur qui s'affiche n'est pas libre, il ne peut donc pas libérer les spectateurs. Au contraire, il travaille, d'une façon consciente ou non, à les conditionner en participant à un mode d'échange culturel dominé par l'économie et dont le film n'est qu'un produit culturel, une valeur marchande de plus parmi tant d'autres. Toute action médiatrice risque ainsi d'être englobée et confondue avec des visées purement commerciales qui dupent une grande partie du public en lui proposant des denrées culturelles et non des œuvres filmiques. En conformité avec la logique des intérêts dominants du système posthollywoodien, la pratique de ces «médiateurs mercenaires» tend à orienter et à spécialiser la demande cinématographique vers quelques superproductions. Elle contribue, de ce fait même, à limiter la diversité des produits filmiques offerts et, par conséquent, à écarter des salles de cinéma une partie du public potentiel.

Groupe de recherche multimédia «Nexus Inc.» 
1 Par réceptivité selon le contexte cinématographique, nous entendons tout ce qui est extérieurà la réceptivité filmique, c'est-à-dire tout ce qui est programmé en terme de lecture et non pas par le texte filmique proprement dit mais bien par le contexte cinématographique dans lequel se situe ce texte, suivant en cela la division analytique proposée par Christian Metz dans Langage et cinéma (Paris: Larousse, 1971).

Le contexte cinématographique posthollywoodien désigne la période historique du cinéma hollywoodien comprise entre 1973 et aujourd'hui. Cette période se caractérise notamment par le passage des Majors sous l'emprise de conglomérats industriels. Cette mutation structurelle majeure a entraîné une nouvelle organisation au sein de l'industrie du cinéma et une nouvelle conception des films hollywoodiens (cinéma-monde, films-événements, superproductions performantes).

Dans notre typologie du cinéma américain, le «film hollywoodien» de facture éminemment commerciale se différencie, tant du point de vue formel que du point de vue idéologique, du «film américain» et du «film états-unien». Pour un aperçu détaillé de ces sujets, voir notre thèse de doctorat: Le Cinéma-monde posthollywoodien: une industrie culturelle transnationale de l'imaginaire filmique. Une analyse sociopragmatique (Sorbonne Nouvelle, Paris III, 1991).

2 Les lois antitrust, autrement appelées Paramount Decrees, édictées par le gouvernement américain en vue d'enrayer la concentration monopoliste dans l'industrie cinématographique américaine, obligeaient les Majors hollywoodiens à se départir de leurs réseaux de salles pour ne conserver que les secteurs de la production et de la distribution cinématographiques.

3 Nous préférons le terme de «médiation» parce qu'il nous apparaît moins statique et surtout plus volontariste que celui de «relais d'opinion» qui néglige, à notre avis, les divers moyens - et leur portée - pour orienter l'opinion publique.

4 Selon une approche sociologique des mass médias, Jean Cazeneuve définit les médiateurs de la façon suivante: «Les guides d'opinion sont des médiateurs, puisqu'ils façonnent pour autrui la portée des messages diffusés; sont également médiateurs, d'une manière ou d'une autre, tous ceux qui interviennent dans la circulation des messages, tous ceux qui à quelque titre que ce soit représentent le public dans une émission, tous ceux qui renseignent le public sur les émissions et sur les films». In Préface «Les médiateurs, quelle influence et sur quel public?», du livre d'Anne-Marie Laulan, Cinéma, presse et public (Paris: Retz-C.E.P.L., 1974) p. 14-15.

5 On confond généralement publicité et promotion. La publicité (environ 80 
$\%$ du budget) s'adresse au public de masse d'une façon anonyme et s'offre sur un mode quantitatif par matraquage et redondance. La promotion se fait selon un mode qualitatif en vue de susciter la fascination, l'intérêt. Elle s'adresse à un public segmenté de façon personnalisée. Les activités publicitaires et promotionnelles sont incluses dans les frais de distribution et placées sous la supervision des Majors. Leur contribution grandissante au succès d'un film a fait grimper en flèche leurs coûts, responsables en grande partie de l'inflation budgétaire des films.

6 En ce qui concerne les affiches de cinéma, voir Anne-Marie Laulan, op. cit., p. 101-105; pour les extraits télévisés, Philippe J. Maarek, Média et malentendus, cinéma et communication politique (Paris: Édilig, 1986) p. 180-185.

7 Livre blanc de la distribution en France, ( Paris, 1980) p. 34.

8 Par exemple aux USA (comme au Canada ou en Fance), la grande presse urbaine (New-York Times, Washington Post, etc.) consacre dans son édition du week-end un cahier entier réservé aux activités cinématographiques. Toutes les chaînes de télévision (networks) ont également inséré dans leur programmation hebdomadaire une émission traitant du VII ${ }^{e}$ art.

9 Pour un aperçu de ce qui différencie la médiation du critique par rapport à celle de l'analyste ou du théoricien de cinéma, voir notamment ces deux articles dans la revue CinémAction, $\mathrm{n}^{\circ} 47$ (Les Théories du cinéma aujourd'hui): Michel Marie, «La Théorie et la critique face aux média et à l'école», p. 177181 et Jean Collet, «A-t-on jamais demandé à une théorie de marcher!», p. 171176.

10 Ch.-A. Michalet, Le Drôle de drame du cinéma mondial. Une industrie culturelle menacée (Paris: éd. La Découverte, 1987) p. 94.

11 Pour le seul film Batman (1989), 24 compagnies ont signé avec la Warner Bros, 44 contrats pour mettre sur le marché des produits dérivés du film. Cf. Jean-Louis Ginibre, «Hollywood, les grandes manœuvres», in Première, $\mathrm{n}^{\circ}$ 148 (juillet 1989) p. 117.

12 M. Pye et L. Myles, Les Enfants terribles du cinéma américain (ou comment les jeunes cinéastes ont pris le pouvoir à Hollywood), (Lausanne: L'Age d'Homme, 1983)p.128. Selon A. Mattelart, dans Transnationals and the Third World, ces produits auxiliaires de Star Wars auraient généré une recette de $\$ 400$ millions (p. 32).

13 Jean-Louis Ginibre, «Hollywood, les grandes manœuvres», Première , $\mathrm{n}^{\circ}$ 148 (juillet 1989) p. 117-118.

14 Philippe J. Maarek, «One + One», Cinématographe , n70 (septembre 1981) 
15 Certains auteurs francophones utilisent le mot séquel ou «séquelle» pour définir ce fait. Malgré l'aspect ironique de symptôme que révèle le terme - le deuxième film ayant les «séquelles» du premier — nous rejetons ce raccourci linguistique paresseux qui n'apporte aucune précision sémantique.

16 En dépit de l'importance reconnue du phénomène des «films à suites» dans le cinéma hollywoodien contemporain, la documentation approfondie sur le sujet est plutôt limitée. On consultera: A. Fauritte, «Hollywood 70-80: les années fastes» in La Revue du cinéma, $n^{\circ}$ 411(déc. 1985)p. 69-77; R. Bezombes et D. Perrin, «Il était une fois», Cinématographe , n 45 (mars 1979) p. 27-32; B. Krohn, «Hollywood à l'heure du "déjà vu"», Cahiers du cinéma , n 423 (sept. 1989) p. 28-32.

17 On utilise ici comme critère de sélection les films qui ont rapporté plus de $\$ 50$ millions en recette-distributeur sur le marché domestique USA/Canada.

18 Entre 1975 et 1988, l'industrie posthollywoodienne a pondu six moutures de Friday 13th et de Police Academy, cinq films de la série Star Trek, quatre productions de Rocky, Superman, Jaws et Airport, ainsi qu'un grand nombre de trilogies dont Star Wars, Indiana Jones, Omen, Halloween, Amityville Horror, etc. Les tournages de Back to the Future II et III se sont faits simultanément la même année. On nous promet pour bientôt le troisième volet des séries Ghost Busters, Aliens, Exorcist, Beverly Hills Cop.

19 L'analyse historique du cinéma hollywoodien a mis en relief comment, face à son incertitude chronique, l'industrie hollywoodienne s'est constamment rabattue sur le principe de la répétition. À commencer par les «séries standardisées» basées sur une thématique identique qui, au fil du temps, se sont cristallisées en «genres»: films d'aventures, films noirs, peplums, comédies musicales, westerns, films de science-fiction, d'horreur, érotiques, etc., et dont les infimes variations n'étaient rendues possibles qu'à partir de règles strictement répétitives (aux genres). Hollywood s'est contenté (ou senti obligé) de faire évoluer périodiquement la norme qu'à la suite de l'usure des «genres» et de la lassitude du public. Ainsi, que ce soit avec les «genres», les serials ou encore les remakes, le cycle de la répétition par la standardisation est un procédé bien ancré dans la tradition hollywoodienne.

$20 \mathrm{La}$ loi des séries a des conséquences néfastes sur la vitalité de la création cinématographique. Son omniprésence dans les sphères de la production et de l'exploitation réduit toujours un peu plus les limites de la diversité filmique. Elle restreint la créativité en soumettant et en bâillonnant les artistes à une production répétitive. La loi des séries «constitue un phénomène d'autocensure professionnelle très forte, puisque la libre émission des messages nouveaux s'en trouve gênée», Philippe J. Maarek (1986), op. cit., p. 123. 
21 Voir à ce sujet notre thèse doctorale (chap. 10.3: Les «suites filmées»: l'offre au-devant de la consommation), op. cit., p. 464-472.

22 La rentabilité des séries apporte des révélations intéressantes. D'abord, on remarque dans l'ensemble que si les succès filmiques ont tendance à se donner suite comme le veut la logique des séries, les «suites filmées» n'en démontrent pas moins une décroissance progressive des recettes. En pratique, les statistiques d'exploitation indiquent de façon évidente que l'effet bénéfique des séries s'amortit avec le temps (lassitude du public, prolifération des contrefaçons, pastiches, etc.). Toutes les suites filmiques, à l'exception de Rocky $I I I$ et $I V$, ont été moins performantes que le film original de chaque série. Même si le modèle de fabrication a fait ses preuves antérieurement, la répétition sérielle tend aujourd'hui à surévaluer les chances de succès de beaucoup de films-à-suite en négligeant d'élaborer un concept d'ensemble et une stratégie appropriée. Car, en fin de compte, c'est le public qui a le dernier mot: la «dégénérescence» continuelle enregistrée par une «série filmée» est la preuve que si le public est passif, il ne l'est pas autant qu'on voudrait le croire. Voir à ce sujet notre thèse doctorale, op. cit., p. 464-472.

23 Robert B. Ray, A Certain Tendency of the Hollywood Cinema, 1930-1980 (Princeton: Princeton University Press, 1985) p. 261.

24 Cette pratique s'inspire de celle du pilot propre à l'industrie télévisuelle américaine. Le pilot est un téléfilm condensé ( 2 heures) d'une nouvelle série de télévision et présenté dans les semaines qui précèdent la programmation de ladite série afin de tester sa valeur narrative et spectaculaire. Cf. A. Mattelart et al., op. cit., p. 175.

25 Elihu Katz et Paul Lazarsfeld, Personnal Influence (the part played by people in mass communications) (Illinois: Glencoe Free Press, 1964).

26 Les «leaders d'opinion» sont différents des «relais d'opinion» parce qu'ils échangent leurs informations sur une base interpersonnelle, d'individu à individu, tandis que les «relais d'opinion» sont des intermédiaires institutionnels qui s'adressent à une audience, par des moyens médiatiques surtout.

27 En conséquence, le sujet-récepteur de communication est rarement atteint dans son rôle de membre anonyme et isolé d'une société de masse. Sa réception du message et ses opinions sont plutôt «médiatisées» à travers ses interactions personnelles au sein de groupes informels auxquels il appartient. Ce qui relativise la thèse de l'École de Francfort qui voit dans l'industrie culturelle de masse «un instrument d'asservissement de la conscience [qui] interdit la formation d'individus autonomes, indépendants, qui jugent et décident consciemment par eux-mêmes». Th. W. Adorno, «L'Industrie culturelle revisitée», Parachute ("Le privé et le public»), n 48 (1987) p. 48. 
28 Philippe J. Maarek ajoute même que la singularité sociale et culturelle privilégiée des jeunes va non seulement renforcer leur rôle de leader d'opinion naturel mais aussi améliorer leur décodage des messages en rapprochant leurs référents esthétiques aussi bien que sémantiques de ceux des émetteurs de messages cinématographiques. Voir Philippe J. Maarek, Média et malentendus, cinéma et communication politique, op. cit., p. 251.

29 Abraham Moles donne la définition suivante de la rumeur: «Type de message circulant dans une masse sociale à partir des contacts interpersonnels successifs et constitué de fragments disjoints de messages diffusés par des canaux et recomposés par les individus en fonction d'un certain nombre de stéréotypes ou de valeurs, pour acquérir une cohérence explicative». Cf. Abraham Moles, La Communication et les mass média (Paris: CEPL, 1971) p. 674.

\section{OUVRAGES CITÉS}

Debord, Guy. La Société du spectacle, $2^{e}$ éd. Paris: Champ Libre, 1971.

Moles, Abraham. La Communication et les mass média. Paris: CEPL, 1971.

Maarek, Philippe J. Média et malentendus, cinéma et communication politique. Paris: Édilig, 1986.

Mattelart, A. et al. La Culture contre la démocratie? L'audiovisuel à l'heure transnationale. Paris: éd. La Découverte, 1983.

Sorlin, Pierre. Sociologie du cinéma. Paris: Aubier Montaigne, 1977. 